\title{
28 INFORMATION TECHNOLOGY DIFFUSION IN THE JORDANIAN TELECOM INDUSTRY
}

\author{
Ala M. Abu-Samaha \\ Amman University \\ Amman, Jordan \\ Ibrahim Mansi \\ The Arab Academy for Banking \\ and Financial Sciences \\ Amman, Jordan
}

\begin{abstract}
The aim of this paper is to analyze the diffusion of information technologies, applications, and systems in the Jordanian telecommunication industry. The paper introduces changes and challenges facing the public switched telephony network (PSTN) providers in Jordan and a number of strategies to face or even to exploit such changes and challenges to PSTN providers' advantage.
\end{abstract}

Keywords Information technology diffusion, public switched telephony network provider, mobile telephony provider, challenges

\section{INTRODUCTION}

A key driver of unrelenting change in organizations is the ever-increasing rate of information technologies and systems implementation and replacement. The introduction of new information technology applications is often associated with changes to organizational structure, the distribution of power, and user working practices/styles (Clegg et al. 1996; Clegg et al. 1997; Doherty and Perry 2001; Poulymenakou and Holmes 1996; Raymond et al. 1995). IT development and utilization provide insights into the nature

Please use the following format when citing this chapter:

Abu-Samaha, A. M., and Mansi, I., 2007, in IFIP International Federation for Information Processing, Volume 235, Organizational Dynamics of Technology-Based Innovation: Diversifying the Research Agenda, eds. McMaster, T., Wastell, D., Ferneley, E., and DeGross, J. (Boston: Springer), pp. 431-442. 
of the complex relationship between the technology and the organization (Bloomfield and McLean 1996; Zuboff 1988). In its broadest sense, implementation refers to all organizational activities involved in the adoption, management and utilization of an innovation. Specifically, in the realm of information systems, adoption is the process of introducing, developing, and deploying/installing a system within an organizational setting whether intra- or interorganizational (i.e., internal to the organization or across a supply chain). Such a process can be considered as a complex process of deliberate organizational change(s) as many organizations seize the chance to introduce deliberate and reactive changes to the organizational setting entailing structure, processes, tasks, roles, job descriptions, relationships to others, and power structure (Orlikowski and Hofman 1997).

This paper introduces an analysis of future IT diffusion in the Jordanian telecommunication industry. The paper starts with the history of telecommunications in Jordan. The third section introduces Jordan Telecom Group (JTG) as a counter to the environmental changes and challenges facing the public switched telephony network (PSTN) providers in Jordan. The fourth section introduces statistical data to show the size and effect of environmental changes and challenges to PSTN services provision. The fifth section introduces a number of strategies adopted by JTG to face or even to exploit the environmental changes and challenges to PSTN providers' advantage. The paper ends with a number of lessons learned and conclusions.

\section{HISTORY OF TELECOMMUNICATIONS IN JORDAN}

Jordan Telecom $\left(\mathrm{J}^{\top} \mathrm{T}\right)$ is a PSTN provider operating and managing a fixed public telecommunication network that provides local, national, and international fixed telephony services and leased lines. In addition to JT, the Jordanian Telecommunications Regulatory Commission granted a class license in May 2005 to BATELCO Jordan to provide PSTN services in the near future.' JT was the exclusive provider of these services until the end of 2004. In addition to PSTN, JT was granted a license for providing GSM900 public mobile telephony on September 15, 2000, through an affiliate (MobileCom). JT enjoyed dual exclusivity (duopoly) for providing GSM900 public mobile telephony service until the end of 2003 when a third player was granted a third license. ${ }^{2}$

In terms of history, Britain's Cable and Wireless Company assisted the Jordanian Department of Post and Telegraph in developing telecommunication services and international links in 1930. The Telecommunications Corporation (TCC) was established as a government controlled entity responsible for various telecom services, including telephone, telegraph, and telex in 1971. In 1997, TCC was transformed into a government-owned company operating on a commercial basis, as a first step toward privatization, and was then renamed Jordan Telecommunications Company (JTC). A consortium

'From the Telecommunications Regulatory Commission website (http://www.trc.gov.jo/ Static_English/market.shtm; accessed on November 12, 2005).

${ }^{2}$ From the Telecommunications Regulatory Commission website (http://www.trc.gov.jo/ Static_English/telecomss.shtm; accessed on November 12, 2005). 
led by France Telecom and the Arab Bank, the largest independent bank in Jordan, purchased 40 percent of JTC in $2000 .^{3}$

\section{JORDAN TELECOM GROUP}

Jordan Telecom Group (JTG) represents the integrated operations, infrastructure and management of Jordan Telecom (the telephony provider), MobileCom (the mobile telephony provider), Wanadoo (the Internet service provider), and e-Dimension (an electronic content and service provider). Jordan Telecom Group aims to provide a higher quality service and a better added value to customers by combining four operating companies into a single one-stop-shop operator.

\subsection{Jordan Telecom (JT Fixed Retail Business Unit)}

Jordan Telecom, initially established by the government of Jordan, is currently considered as a privately owned public company dominated by France Telecom, the largest shareholder in the company. With more than 600,000 customers, Jordan Telecom's priorities include (1) developing and improving the fixed line telephone network, (2) extending the reach of the national data transmission network to ensure that every citizen/customer has the opportunity of being globally connected, and (3) lowering telephony tariffs.

\subsection{MobileCom (JT Mobile Business Unit)}

MobileCom, a subsidiary of Jordan Telecom, is one of four mobile telephone service providers in Jordan. The company was established in September 1999 with the aim of building a new, highly advanced mobile communications network. MobileCom is committed to continually introducing new levels of customer service, satisfaction, and quality, enabling Jordanians to benefit from the advantages of mobile communication. By coupling advanced technology and quality with value for money services, today MobileCom has more than 1,000,000 customers and a GSM network coverage reaching over 99 percent of the Jordanian population.

\subsection{Wanadoo (JT Internet and Data Business Unit)}

Previously known as Global One and Equant, Wanadoo emerged on the Jordanian market in 2001 following a strategic partnership with France Telecom. Wanadoo, Europe's largest Internet service provider, is leading the Internet service provision (ISP) market in Jordan with more than 50 percent of market share. Wanadoo continues to lead the Jordanian ISP market through introducing first-to-market services such as ADSL (asymmetric digital subscriber line), Cool Net Pre-Paid Internet Cards, Internet accessibility via 0900 service, full-fledged data communication and Internet roaming, to name just a few.

${ }^{3}$ Ibid. 


\section{4 e-Dimension (JT Content Business Unit)}

Inaugurated in 2002 to address the needs of JT Group and the local/regional markets, eDimension, a de facto industry standard, is a leader in the IT solutions and content provision markets with an array of products including web software solutions development, ASP (application service provider), ICDN (Internet content delivery network) technologies, integration, specialized high-end PSP (payment service provider), and IPTV and triple play content provision. Exploiting the expertise of its highly experienced and internationally recognized team, e-Dimension successfully launched Jordan's first and only e-Payment gateway in 2003. e-Dimension was the first to develop and integrate an online real-time rating and billing engine for $\mathrm{J}^{\top} \mathrm{T}$ Double Play. e-Dimension integrated and provisioned the SVI/IVR and Saga800 virtual call centers for JT, which enables operatorclass deployment of any combination of human resources at any location for customer interaction. e-Dimension has recently partnered with local, regional and international content providers to pave the way for developing the infrastructure for triple play and IPTV services, which offers a combination of voice, data, and video to be launched soon.

\subsection{The Wholesale Business Unit}

The wholesale business unit was created to address the business requirements of the existing and newly licensed service providers in a consistent, regulatory compliant, and time effective manner. The significant strategic partnership with France Telecom has enabled the Group to open up wider perspectives with new wholesale services, new technologies, and a worldwide presence.

\section{CHALLENGES TO FIXED LINE PENETRATION}

Public switched telephony network providers face serious challenges from the mobile and Internet service providers, mainly the high penetration rate of mobile devices compared to the decreasing penetration rate of fixed lines and the accelerated move to voice over IP services.

In terms of national infrastructure, Jordan has one telecommunication company (JT), four mobile network operators mostly owned by the private sector, and an extended number of ISPS, all of which are owned and operated by the private sector. In order to meet the increasing demand for high quality, high bandwidth Internet connectivity, the government of Jordan has issued 21 additional ISP licenses. ${ }^{4}$ In addition, the Telecommunications Regulatory Commission also granted a class license to BATELCO Jordan May 2005 to provide PSTN services in the near future to the Jordanian market and a class license to Thuraya Satellite Telecommunications Company to provide global mobile personal communications by satellite (GMPCS). ${ }^{5}$

${ }^{4}$ For information on ISP licenses in Jordan, see the Telecommunications Regulatory Commission's website (specifically, http://www.trc.gov.jo/Static_English/market.shtm) and Arab Advisors Press Room (http://www arabadvisors.com/Pressers/presser-181005.htm).

${ }^{5}$ From the Telecommunications Regulatory Commission's website (specifically, http:// www.trc.gov.jo/Static_English/telecomss.shtm). 
Jordan's cellular subscribers grew at a rate of 46.8 percent between 2000 and 2004 . Reduced rates, per second billing, extended validities and special offers, were expected to cause the market to grow at a rate of 46 percent in 2005. Between 2006 and 2009, the Arab Advisors Group expects the Jordanian cellular market to grow at a rate of 10 percent, to exceed 3.43 million subscribers by 2009 ; with a penetration rate of more than 57 percent. ${ }^{6}$ The number of fixed line/PSTN subscribers decreased from 629,000 in 2002 to 623,000 in 2004 and currently is estimated at 600,000 . In the same period of time, the number of mobile subscribers rose to $1,624,000$ in 2004 from 1,219,000 in 2002. ${ }^{7}$ AbuGhazaleh \& Co. Consulting (2005) estimated the penetration rate of cellular/mobile phone at 30.9 percent in 2004, with a total number of mobile owner population of 1,801,100. Regarding Internet accessibility, on the other hand, Abu-Ghazaleh and Co. estimated the number of Internet users in Jordan at 111,054 in 2004, up from 62,242 in 2002.

\section{INFORMATION TECHNOLOGY DIFFUSION IN JORDAN TELECOM}

Basically, telecommunication technologies were developed to transfer voice. Initially such transmission was achieved through analog systems based on microwave technology, which were limited in capacity. The disadvantages of the analog transmission and switching system pushed developers and technology manufacturers to look for more enhanced and effective telecommunication systems based on PCM (pulse code modulation). PCM is a digital signal of $2048 \mathrm{~Kb} / \mathrm{s}$ speed that can transfer up to 30 voice channels. The need for enhanced channel capacity for data and voice transmission during the late 1980s resulted in PDH (plesynchronous digital hierarchy) followed with SDH (synchronous digital hierarchy). This higher technology capacity matches the needs for data transmission caused by increased Internet traffic. Initially, Internet connectivity was available via dial up connections to pass through digital circuit switches which provide limited bandwidth, continuous billing during connectivity, and a busy analog telephone set. In order to meet the even higher needs for Internet connectivity, ADSL (asymmetrical digital subscriber line) over soft switch was introduced to provide wireless connectivity. By the year 2012, all telecommunication media (voice, data, and video) will be integrated through soft switch by using complete packet switching technology. By doing so, JTG as a telecommunication operator will provide its customers all the services needed through one platform, which will lead to reduced service cost, the possibility of increasing the bandwidth needed by customers, and interconnection with international operators to enhance services provision globally via satellite between JTG and France Telecom.

In order to absorb the accelerating move to IP-based services and the ever-increasing challenge from mobile telephony, the industry's network management paradigm is rapidly shifting from traditional infrastructure management to the management of next-

${ }^{\dagger}$ See Arab Advisors Press Room, http://www arabadvisors.com/Pressers/presser-181005.htm.

${ }^{7}$ Information taken from Department of Statistics website (www.dos.gov/jo; accessed November 12, 2005). 


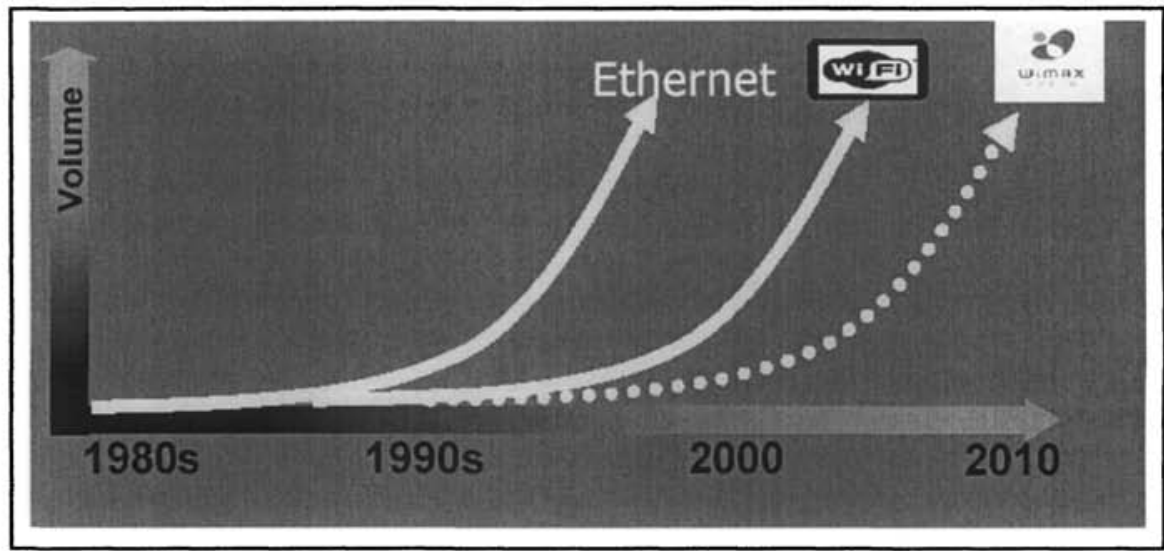

Figure 1. Jordan Telecom Technology Changes

generation, converged infrastructure (bearing both traditional and packet traffic) and the revenue-generating component of network services. While network element and domain monitoring remain critically important, today's quality of service commitments require a clear, real-time view of traffic and performance to ensure services are delivered end-toend to the customer. Figure 1 depicts the JT technology changes reaching the vision of 2010.

Currently, JT is considering three major emerging technologies to be included within its current suit of services and products; these are voice over IP (VoIP), WiFi and WiMax. The costs of ownership and life cycle are posing important embodiments for implementing these technologies. The following sections provide a brief overview of these technologies and their JT Group implementation.

\subsection{Voice Over IP}

Protocols which are used to carry voice signals over the IP network are commonly referred to as voice over IP or VoIP protocols. Some cost savings are due to utilizing a single network to carry voice and data, especially where users have existing, underutilized network capacity. VoIP to VoIP phone calls are typically free, while VoIP to PSTN calls generally require an added cost to the VoIP user. VoIP is gaining an advantage over PSTN, replacing the regular switching with new soft IP-based switches and it is generally under-implemented in JT Group. VoIP offers features and services that are not available via traditional phones. Where there is a broadband Internet connection, there is no need to maintain and pay the additional cost for a line just to make telephone calls. Figure 2 provides a diagrammatic representation of the VoIP environment.

\subsection{WiFi Network Technology in JT Group}

WiFi (wireless fidelity) is a data transmission system designed to provide location independent network access between computing devices by using radio waves rather than a 


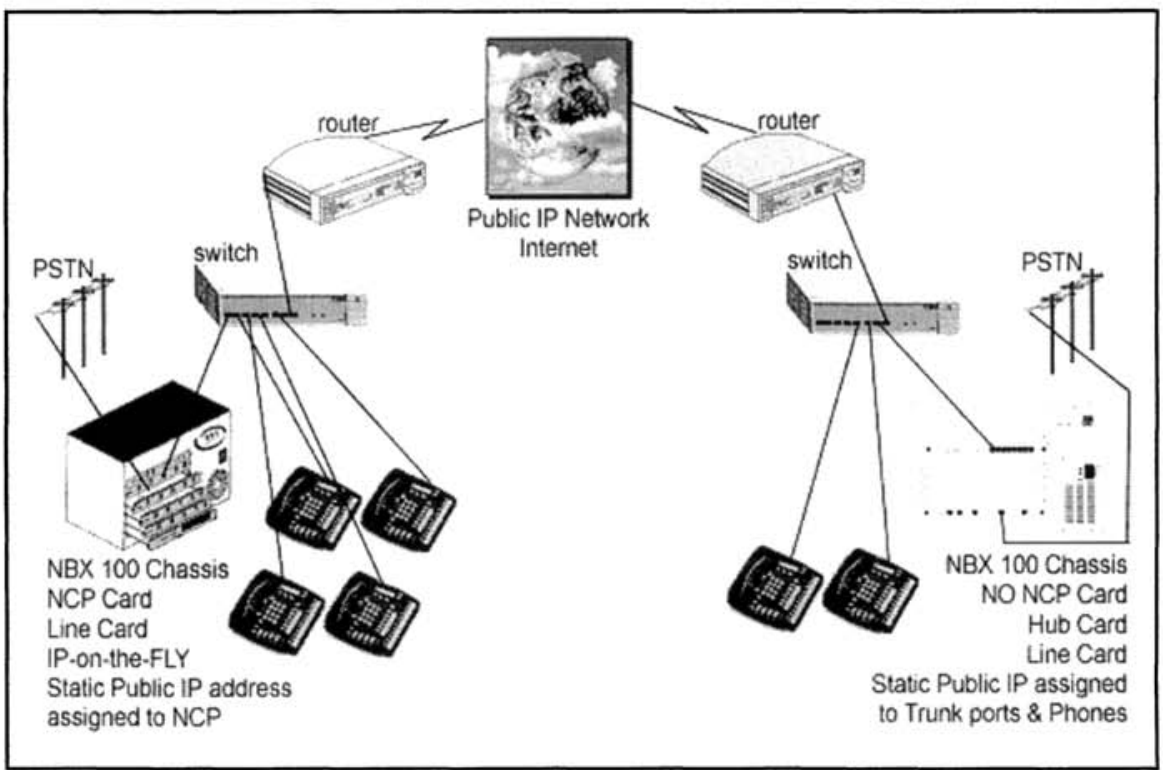

Figure 2. Voice over IP in Jordon Telecom Group

cable infrastructure. In the corporate enterprise, wireless LANs are usually implemented as the final link between the existing wired network and a group of client's computers, giving these users wireless access to the full resources and services of the corporate network across a building or campus setting. ${ }^{8}$ Figure 3 provides a diagrammatic representation of a WiFi environment.

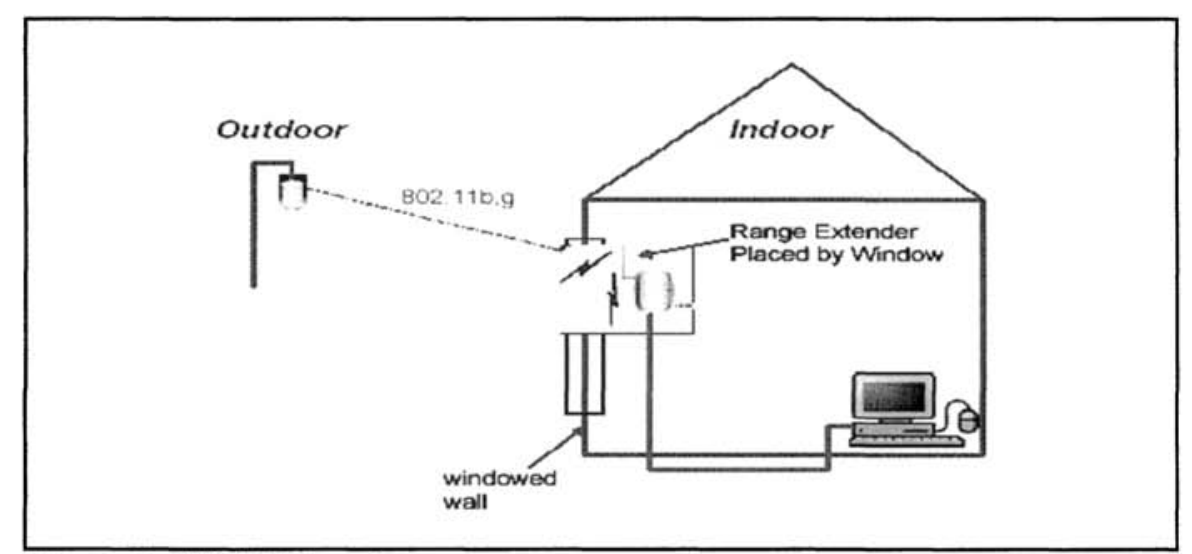

Figure 3. WiFi Network

${ }^{8}$ From the TelecomSpace web site, http://www.telecomspace.com/wirelessnw-wifi.html, accessed on January 10,2006. 
WiFi has many advantages over other alternative technologies; these can be summarized as follows:

- Unlike packet radio systems, WiFi uses unlicenced radio spectrum and does not require regulatory approval for individual deployments.

- WiFi allows LANs to be deployed without cabling, potentially reducing the costs of network deployment and expansion. Spaces where cables cannot be run, such as outdoor areas and historical buildings can host wireless LANs.

- WiFi networks support roaming, in which a mobile client station such as a laptop computer can move from one access point to another as the user moves around a building or area.

- Many access points and network interfaces support various degrees of encryption to protect traffic from interception.

- Unlike cellular carriers, the same WiFi client works in different countries around the world. ${ }^{9}$

\subsection{WiMax Network Technology in JT Group}

WiMax is a worldwide interoperability for microwave access allowing broadband wireless access of information in the form of data packet. Figure 4 describes the JT Group WiMax trial.

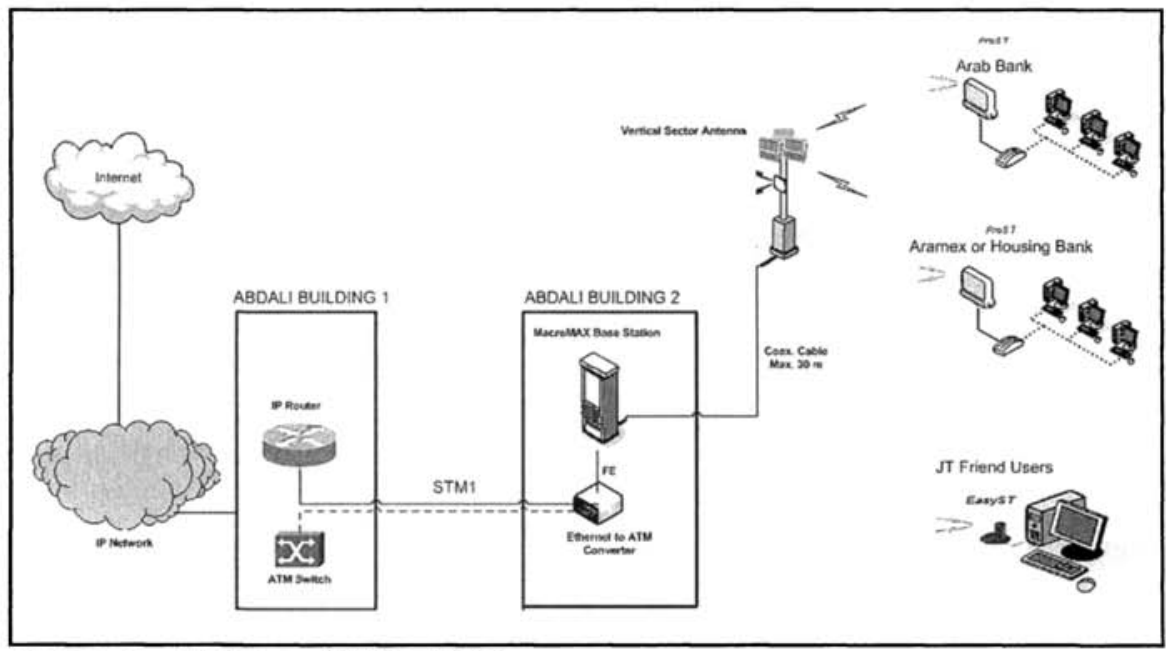

Figure 4. JT Group WiMax Trial

${ }^{9}$ Ibid. 
WiMax is a technology that delivers last mile broadband connectivity in a larger geographic area than WiFi, enabling service to business customers and cable DSLequivalent access to residential users providing coverage anywhere from 1 to $10 \mathrm{~km}$. WiMax will enable greater mobility, range, and throughput for high speed data applications. The motivators for WiMax are

- low-cost high-performance solution to deliver broadband wireless data

- new business opportunities for broadband services reaching developed, emerging, and rural markets

- designed to operate as a complementary network to the third generation technologies

\subsection{The NMC Project}

In addition to the technologies mentioned, JTG launched the Jordan Telecom Group Network Centre (NMC) to revolutoinize the way the company manages its infrastructure. The Jordon Telecom Group NMC project came to meet many objectives, which motivated the movement tothe next generation of network management. These are

- increasing revenue through increasing the call completion rate

- reducing operating cost through improved operations and maintenance of the network

- making better use of people (rightsizing not downsizing, too high staff/line ratio)

- meeting regulatory standards

- minimizing service restoration time

- improving quality of the network and services

- managing the resources and reducing duplication of resources

- better informing the customers

- centralizing the work in one location

- managing and coordinating fieldwork through resources management and good field team piloting and handling of faults

- identifying cause, effect and status of system failures

- preparing for deregulation

In order to realize the objectives of the NMC project, NetBoss was selected by JTC to deliver reliable, flexible network management systems to commercial, government, and utility communications providers globally for the next a decade. NetBoss Network Management focuses on the service assurance and service fulfilment needs of complex, multi-vendor, multi-protocol network operators. A full-featured suite of applications covering real-time fault management, performance management, service activation, billing mediation, e-bonding, and enterprise application integration is available for a broad range of network operators - from telecommunications service providers to large private networks. NetBoss manages everything from legacy to next-generation, IP-based network infrastructure and services, offering a scalable, future-proof solution for convergent service providers. This movement and convergence of fault and performance management enables the operators to truly plan and manage service quality and proactively correct problems before they significantly impact the customers, which will increase the 
company performance and reliability. ${ }^{10}$ NetBoss supported the major goals of the JTGNMC project and is one of the best solutions for network management. NetBoss is an integrated communications management platform that supports wireless, wire line, and Internet services.

The strategic benefits of NetBoss include lower life cycle costs, maximization of the existing investment; reduced IT and administration costs; bundled solution pricing; and faster delivery of new services. Operational operational benefits include rapid deployment; improved network and service reliability; redundant and scalable solution; multivendor, multi-service, multi-protocol solutions; an open architecture; and single vendor for faster response. The added values for this movement include

- Flexible, scalable, proven solution: Regardless of the equipment and service mix in the network, NetBoss offers powerful element, shelf, rack, segment, and full network views, as well as fault management, proactive network control, database and application monitoring and automated processes.

- Trouble ticketing: NetBoss is already integrated with a powerful alarm reporting system, which is a dynamic tool to automatically assign a given alarm at the NetBoss fault management system screen to the related team.

- Service management quality and service level management: As the new economic models have evaporated and management attention has returned to fundamentals, there has been a corresponding increase in the level of discussion surrounding the concept of service level management. While there is much discussion about the need to invest significant resources in new and sophisticated applications, service providers can leverage existing resources significantly by building relatively simple, integrated service-level views of their networks. These views provide network managers and customer account managers with the information they need to proactively work with key clients while addressing network infrastructure issues on a priority basis."

Figure 5 shows the NMC work flowchart.

\section{CONCLUSIONS}

This paper has produced an analysis of the major challenges that face the Jordanian PSTN market and few of the strategies undertaken by the sole PSTN provider to counter these challenges. The paper has provided a number of statistics to show the severe migration from traditional fixed accessibility to mobile and Internet accessibility. The paper presented three communication technologies - voice over IP, WiFi, and WiMaxto be included within the current suit of services and products offered by PSTN providers. This new mix of products is expected to recapture the telecommunication market by Jordan Telecom Group that was lost over the past few years to mobile telephony operators and ISPs.

${ }^{10}$ From the NetBoss Manual (http://www.networksupport.harris.com/support/documents/ 1000.pdf; accessed January 10, 2006).

"Ibid. 


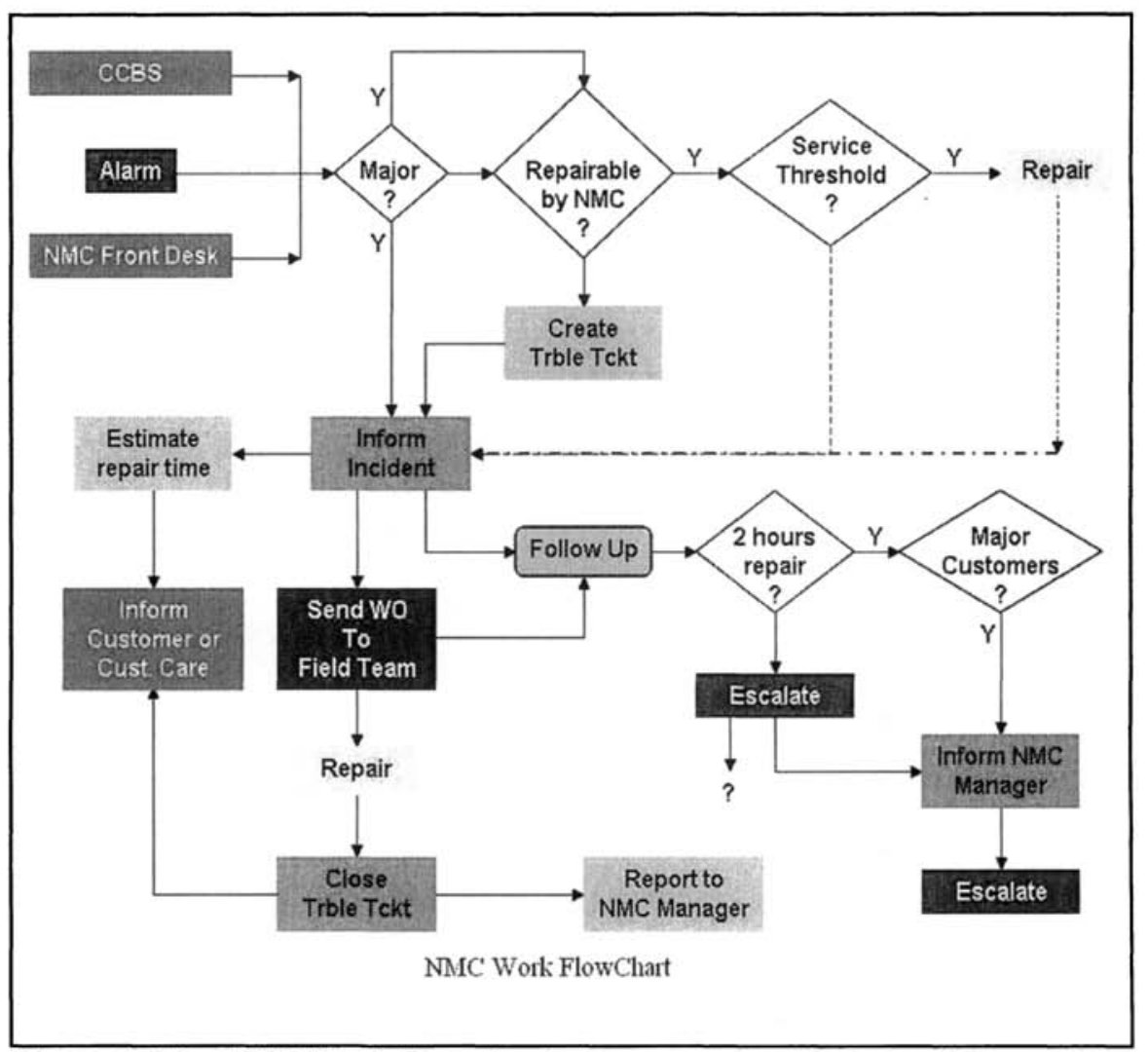

Figure 5. The NMC Work Flowchart

\section{References}

Abu-Ghazaleh and Co. Consulting. "Market Brief on Telecommunications Sector in Jordan," 2005 .

Bloomfield, B. P., and McLean, C. "Madness and Organization: Informed Management and Empowerment," in W. J. Orlikowski, G. Walsham, M. R. Jones, and J. I. DeGross (eds.), Information Technology and Changes in Organizational Work, London: Chapman \& Hall, 1996, pp. 371-393.

Clegg, C., Axtell, C., Damadoran, L., Farbey, B., Hull, R., Lloyd-Jones, R., Nicholls, J. Sell, R., and Tomlinson, C. "Information Technology: A Study of Performance and the Role of Human and Organizational Factors," Ergonomics (40:9), 1997, pp. 851-871.

Clegg, C., Coleman, P., Hornby, P., McClaren, R., Robson, J., Carey, N., and Symon, G. "Tools to Incorporate Some Psychological and Organizational Issues During the Development of Computer-Based Systems," Ergonomics (39:3), 1996, pp. 482-511.

Doherty, N. F., and Perry, I. "The Cultural Impact of Workflow Management Systems in the Financial Services Sector," Service Industries Journal (21:4), 2001, pp. 147-166.

Orlikowski, W. J., and Hofman, D. "An Improvisational Model of Change Management: The Case of Groupware Technologies," Sloan Management Review (38:2), 1997, pp. 11-21. 
Poulymenakou, A., and Holmes, A. "A Contingency Framework for the Investigation of Information Systems Failure," European Journal of Information Systems (5), 1996, pp. 34-46.

Raymond, L., Pare, G., and Bergeron, F. "Matching Information Technology and Organizational Structure: An Empirical Study with Implications for Performance," European Journal of Information Systems (4), 1995, pp. 3-16.

Zuboff, S. Dilemmas of Transformation in the Age of the Smart Machine, New York: Basic Books, 1988.

\section{About the Authors}

Ala M. Abu-Samaha is an assistant professor of Information Systems and head of the Computer Information Systems Department at the Faculty of Information Technology, University of Amman, Jordan. He has contributed to two major areas of research in the information systems discipline: information systems development methodologies and information systems/technology evaluation. Ala holds a Ph.D. in Information Systems from the University of Salford in the UK. He can be reached by e-mail at ala_samaha@yanoo.com.

Ibrahim Mansi is a doctoral student in MIS at the Arab Academy for Banking and Financial Sciences, in Amman, Jordan. He received his Master's in Public Administration from the University of Jordan in 2003, a B.Sc. in Public Administration from Mu'ta University (Jordan) in 2000 , and a diploma in Technical Engineering in 1994. He has been a technical and managerial employee in Jordan Telecom Group since 1995. Ibrahim can be reached by e-mail at imansi@jordantelecomgroup.jo. 